OPEN ACCESS

Edited by:

Elisa Baratella,

University of Trieste, Italy

Reviewed by:

Mengqi Jiang,

University of Maryland, Baltimore,

United States

Mattia Bellan,

Università del Piemonte Orientale, Italy

*Correspondence:

Qi-Jun Wu

wuqj@sj-hospital.org

Ting-Ting Gong

gongtt@sj-hospital.org

${ }^{\dagger}$ These authors have contributed equally to this work

Specialty section: This article was submitted to

Pulmonary Medicine,

a section of the journal

Frontiers in Medicine

Received: 12 November 2021 Accepted: 27 December 2021

Published: 20 January 2022

Citation:

Gao C, Guo J, Gong T-T, LV J-L, Li X-Y, Liu F-H, Zhang M, Shan Y-T, Zhao Y-H and Wu Q-J (2022) Sleep

Duration/Quality With Health Outcomes: An Umbrella Review of

Meta-Analyses of Prospective Studies. Front. Med. 8:813943. doi: 10.3389/fmed.2021.813943

\section{Sleep Duration/Quality With Health Outcomes: An Umbrella Review of Meta-Analyses of Prospective Studies}

\author{
Chang Gao ${ }^{1,2 t}$, Jiao Guo ${ }^{3 \dagger}$, Ting-Ting Gong ${ }^{4 *}$, Jia-Le Lv ${ }^{1,2}$, Xin-Yu Li ${ }^{1,2}$, Fang-Hua Liu ${ }^{1,2}$, \\ Meng Zhang ${ }^{1,2}$, Yi-Tong Shan ${ }^{5}$, Yu-Hong Zhao ${ }^{1,2}$ and Qi-Jun Wu ${ }^{1,2 *}$ \\ ${ }^{1}$ Clinical Research Center, Shengjing Hospital of China Medical University, Shenyang, China, ${ }^{2}$ Department of Clinical \\ Epidemiology, Shengjing Hospital of China Medical University, Shenyang, China, ${ }^{3}$ Department of Oncology, Shengjing \\ Hospital of China Medical University, Shenyang, China, ${ }^{4}$ Department of Obstetrics and Gynecology, Shengjing Hospital of \\ China Medical University, Shenyang, China, ${ }^{5}$ Department of Statistics, University of Washington, Seattle, WA, United States
}

Background: To quantitatively evaluate the evidence of duration and quality of sleep as measured by multiple health outcomes.

Methods: This review is registered with PROSPERO, number CRD42021235587. We systematically searched three databases from inception until November 15, 2020. For each meta-analysis, the summary effect size using fixed and random effects models, the 95\% confidence interval, and the 95\% prediction interval were assessed; heterogeneity, evidence of small-study effects, and excess significance bias were also estimated. According to the above metrics, we evaluated the credibility of each association.

Results: A total of 85 meta-analyses with 36 health outcomes were included in the study. We observed highly suggestive evidence for an association between long sleep and an increased risk of all-cause mortality. Moreover, suggestive evidence supported the associations between long sleep and 5 increased risk of health outcomes (stroke, dyslipidaemia, mortality of coronary heart disease, stroke mortality, and the development or death of stroke); short sleep and increased risk of overweight and/or obesity; poor sleep quality and increased risk of diabetes mellitus and gestational diabetes mellitus.

Conclusions: Only the evidence of the association of long sleep with an increased risk of all-cause mortality was graded as highly suggestive. Additional studies are needed to be conducted.

Systematic Review Registration: https://www.crd.york.ac.uk/PROSPERO/, identifier: CRD42021235587

Keywords: health outcomes, meta-analysis, sleep duration, sleep quality, umbrella review

\section{INTRODUCTION}

Sleep is an important and complex physiological process for maintaining optimal health. The National Sleep Foundation recommends 7-9 h of sleep for people aged 26-64 years and 7-8 h of sleep for people aged $\geq 65$ years (1). However, because of irregular working, shift-work patterns, and unhealthy sleeping habits, the quantity and quality of sleep may be abnormal in modern society. Over the last few decades, there has been growing evidence to suggest that self-reported short or long sleep duration (often defined as $<6$ or 7 and $>8$ or $9 \mathrm{~h}$, respectively) and poor sleep quality 
[Pittsburgh Sleep Quality Index (PSQI) > 5] may be consistently associated with adverse health outcomes [e.g., short sleep and increased risk of hypertension (2), long sleep and increased risk of chronic kidney disease (3), poor sleep quality and increased risk of preterm birth (4), etc.].

Although previous studies have examined this topic using various methodologies, a quantitative appraisal of epidemiological credibility is lacking, as are examinations of the potential bias between the quantity and quality of sleep and health-related outcomes and assessments of the most influential outcomes. Therefore, in the present study, we conducted an umbrella review of the evidence between the quantity and quality of sleep and the multiple health outcomes in systematic reviews and meta-analyses, assessed the diverse bias, and quantitatively evaluated the strength and credibility of the evidence.

\section{METHODS}

We strictly followed standardized guidelines to perform an umbrella review, which is the systematic collection and evaluation of multiple systematic reviews and meta-analyses conducted on a specific research topic (5). The umbrella review was conducted according to the Preferred Reporting Items for Systematic Reviews, Meta-Analyses guidelines (Supplementary Table S1) (6), and the guidance of the Meta-analysis of Observational Studies in Epidemiology statement (Supplementary Table S2) (7). The study protocol was registered in the PROSPERO database for systematic reviews and meta-analyses (registration number: CRD42021235587).

\section{Search Strategy}

The electronic databases, PubMed, EMBASE, and the Web of Science were searched systematically from inception until November 15, 2020, to identify related systematic reviews and meta-analyses of observational studies. A predefined search strategy was used, which is presented in Supplementary Table S3. In addition, we performed a manual check of reference lists from the retrieved articles for further potentially relevant articles.

\section{Eligibility Criteria and Appraisal of Included Studies}

Two authors (CG and X-YL) independently scrutinized articles based on titles and abstracts. If needed, full articles were retrieved for a final decision. Disagreements between the two reviewers were resolved by discussion and consensus with a senior advisor (T-TG). Articles were included according to the following criteria: (A) systematic reviews and meta-analyses of prospective studies on the associations between duration and quality of sleep and any health-related outcome, (B) studies that relied on data from human studies with any type of health-related outcome measure, and (C) studies that reported effect sizes such as odds ratios (ORs), relative risk (RRs), or hazard ratios (HRs) at follow-up. We included information that we were interested in in each study, such as subgroup analysis and dose-response analysis. If a systematic review or meta-analysis performed a subgroup analysis stratified by the study design, then the results for prospective studies were included (8-10).

We excluded individual studies according to the following criteria: (A) meta-analyses of case-control or cross-sectional studies, (B) studies in which sleep measures were not the exposure of interest (such as sleep-disordered breathing, restless leg syndrome, or napping), (C) meta-analyses or systematic reviews that did not present study-specific data [effect sizes, 95\% confidence intervals (CIs) and numbers of cases/population)], (D) systematic reviews without a quantitative synthesis, or (E) other types of papers (e.g., review, abstract, non-English, or editorial). For the main analysis, whenever an eligible meta-analysis included a lower number of component studies compared to other meta-analyses related to the same association, we retained the one with the largest number of primary studies (8-10).

\section{Exposure Identification}

For exposures, the studies of sleep duration included "short sleep duration" and "long sleep duration." In most studies, "sleep duration" was defined as hours per day or minutes per night. "Short sleep duration" was defined as $\leq 5,<5, \leq 6,<6, \leq 5-6$ or $<7$, and "long sleep duration" was defined as $>7, \geq 8, \geq 9$ or $\geq 8$ $9 \mathrm{~h}$. The reference categories for sleep duration in the studies, in $\mathrm{h}$ per night, were $7,7-8,6-8$ or $7-9 \mathrm{~h}$. In this umbrella review, "poor sleep quality," "good sleep quality" and "not-poor sleep quality" were separately characterized as PSQI $>5$, PSQI $<5$ and $\mathrm{PSQI} \leq 5$.

\section{Data Extraction}

Two investigators (X-YL and F-HL) independently extracted the related data from the included studies using a custom-made data extraction form. In the case of discrepancies, the data were subsequently verified by a third author (CG). The data-collection form included the first author, year of publication, journal of publication, exposure, outcome examined, number of included studies, case number, and study population. For each of the included studies in each eligible meta-analysis, we extracted the first author, year of publication, epidemiological design, number of cases and total population, and the maximally adjusted relative risk (ORs, RRs or HRs) along with the corresponding 95\% CI.

\section{Data Analysis}

For each exposure and outcome pair, we evaluated the summary effect size and the 95\% CI through both fixed and random effects models $(11,12)$. The heterogeneity between studies was assessed with the $\mathrm{I}^{2}$ metric of inconsistency and its $95 \% \mathrm{CI}$ (13). The $\mathrm{I}^{2}$ ranges between 0 and $100 \%$ and quantifies the variability in effect estimates that it is due to heterogeneity rather than sampling error. Values exceeding 50\% were indicative of high heterogeneity, whereas values $>75 \%$ implied very high heterogeneity (14). We also calculated the $95 \%$ prediction interval (PI), which further accounted for heterogeneity between studies and estimated the uncertainty of the association if future studies examine that same association (15). 
We used Egger's regression asymmetry test to identify smallstudy effects (16) to evaluate whether smaller studies tend to give higher risk estimates compared with larger studies, which can indicate publication, other reporting biases, or other reasons for differences between small and large studies (17). We calculated the standard error of the effect size for the largest data set of each meta-analysis to determine whether larger estimates of effect size were predicted by small studies compared to large studies (10). Indication of small study effects was based on the $P$ value for Egger's test was smaller than 0.10 and the largest study had a smaller effect size than the summary effect size (17).

We applied the excess significance test to evaluate whether the observed number of studies $(\mathrm{O})$ with statistically significant results among those included in a meta-analysis was larger than the expected number of studies (E) with statistically significant results (18). E is calculated by the sum of the statistical power estimates for each component study. The statistical power of each study was calculated with an algorithm using a noncentral $t$ distribution (19). The excess significance test for single meta-analyses was considered positive at $P<0.10$, given that $\mathrm{O}>\mathrm{E}$ as previously proposed (10). When standardized mean differences were reported, we planned to transform these estimates into ORs (20). The statistical analysis and the power calculations were conducted in STATA version 15.0 , and all $P$ values were two-tailed.

\section{Methodological Quality Appraisal}

To study the quality of the reporting of the included systematic reviews and meta-analyses, two investigators (CG and $\mathrm{X}$ YL) independently rated the methodological quality with the Assessment of Multiple Systematic Reviews (AMSTAR-1) tool. Higher scores imply greater quality, ranging from 0 to 11 . The AMSTAR-1 tool involves dichotomous scoring ( 0 or 1 ) of 11 related items to assess the methodological rigor of the included articles, such as a comprehensive search strategy or publication bias assessment. AMSTAR-1 scores are graded as high (8-11), medium (4-7), and low quality (0-3) (21).

\section{Grading the Evidence}

Statistically significant meta-analyses $(P<0.05)$ were rated into four levels (convincing, highly suggestive, suggestive, and weak) using specific criteria. For convincing evidence: $P<10^{-6}$, number of cases $>1,000, \mathrm{I}^{2}<50 \%, P<0.05$ of the largest component study in the meta-analysis, 95\% PI excludes the null value, absence of small-study effects $(P>0.1$ for Egger's test), and no excess significance bias $(P>0.1)$. For highly suggestive evidence: $P<10^{-6}$, number of cases $>1,000$, and $P<0.05$ of the largest study. For suggestive evidence: $P<10^{-3}$, and number of cases $>1,000$. For weak evidence, the sole criterion was $P<0.05$ (22). When $P>0.05$, there was no association (10). All analyses were conducted in STATA, version 15.0.

\section{RESULTS}

\section{Study Selection}

As reported in Figure 1, 15,669 records were retrieved across three electronic databases search, and 7,958 records were identified unduplicated through the parallel reviews. A total of 7,728 records were excluded after title and abstract screening, and 201 were excluded through assessment of the full-text (Figure 1). Ultimately, 36 articles were included in our umbrella review for analysis.

\section{Characteristics of Included Meta-Analyses}

The characteristics of these 36 articles are summarized in Table 1. All articles were published between 2009 and 2020. These included studies covered 85 meta-analyses, which reported associations between duration and quality of sleep and 36 different outcomes. The median number of original studies in each meta-analysis was 7 (range from 3 to 27), while the median number of cases was 4,848 (range from 156 to 219,518), and the median number of the total participants was 113,226 (range from $1,230$ to $2,311,390)$. The case number exceeded 1,000 in 81 meta-analyses.

\section{Summary Effect Size}

Of the 78 meta-analyses from 32 articles regarding sleep duration, the summary random effects estimates were significant at $P<1 \times 10^{-6}$ in 10 (13\%) meta-analyses, and the summary fixed effects estimates were significant in 28 (36\%) metaanalyses (Supplementary Table S4). Thirty-nine (50\%) metaanalyses reported that the largest study effect was nominally statistically significant, with a $P<0.05$, and a more conservative effect than the summary random effects was observed in 57 (73\%) meta-analyses. The studies with the smallest SE for each association suggested that 30 of 78 were significant at $P<0.05$.

Out of the 7 meta-analyses from 5 articles regarding sleep quality, the summary fixed-effects and random-effects estimates were significant at $P<0.05$. However, when we used $(P<1$ $\left.\times 10^{-6}\right)$ as a threshold for significance, the summary random effects estimates were not significant in any meta-analyses, and $3(43 \%)$ meta-analyses produced significant summary results using the fixed-effects methods (Supplementary Table S4). The studies with the smallest standard error for each association suggested that 5 of 7 were significant at $P<0.05$, and a more conservative effect than the summary random effects was observed in 5 (71\%) meta-analyses. The studies with the smallest SE for each association suggested that two of 7 were significant at $P<0.05$.

\section{Heterogeneity and Prediction Intervals}

In all the included studies regarding sleep duration, approximately $44(56 \%)$ studies had a lower heterogeneity, with $\mathrm{I}^{2} \leq 50 \%$; approximately $28(36 \%)$ studies had substantial heterogeneity estimates, with $\mathrm{I}^{2}$ between 50 and $75 \%$; and 6 (8\%) studies had considerable heterogeneity estimates, with $\mathrm{I}^{2}>75 \%$ (Table 2). When 95\% PIs were evaluated, we found that 10 meta-analyses [cognitive disorders, stroke, diabetes, all cancer mortality, all-cause mortality, mortality (both all-cause and cause-specific), stroke mortality, obesity, overweight and/or obesity, and the development or death of stroke] excluded the null value (Table 2 ). 


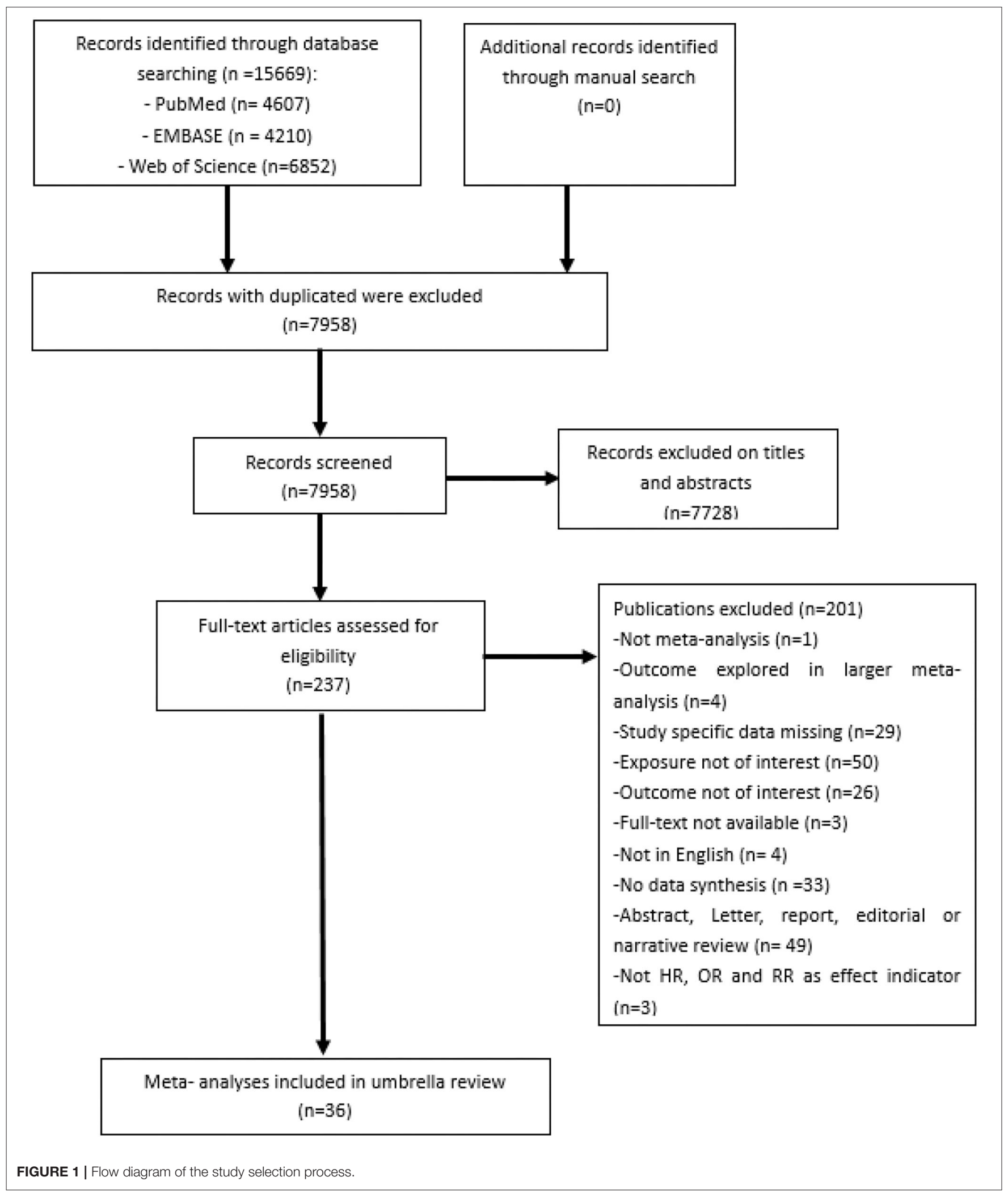

Of the 7 meta-analyses about sleep quality, 3 (42\%) showed low heterogeneity $\left(\mathrm{I}^{2}<50 \%\right)$, and $2(29 \%)$ separately showed substantial heterogeneity $\left(\mathrm{I}^{2}>50 \%\right.$ and $\left.\mathrm{I}^{2} \leq 75 \%\right)$ and considerable heterogeneity $\left(\mathrm{I}^{2}>75 \%\right.$; Table 2$)$. When $95 \%$ PIs were evaluated, we found that no meta-analysis excluded the null value (Table 2). 
TABLE 1 | Main characteristics of included systematic reviews or meta-analyses that evaluate sleep duration/quality and health outcome risk.

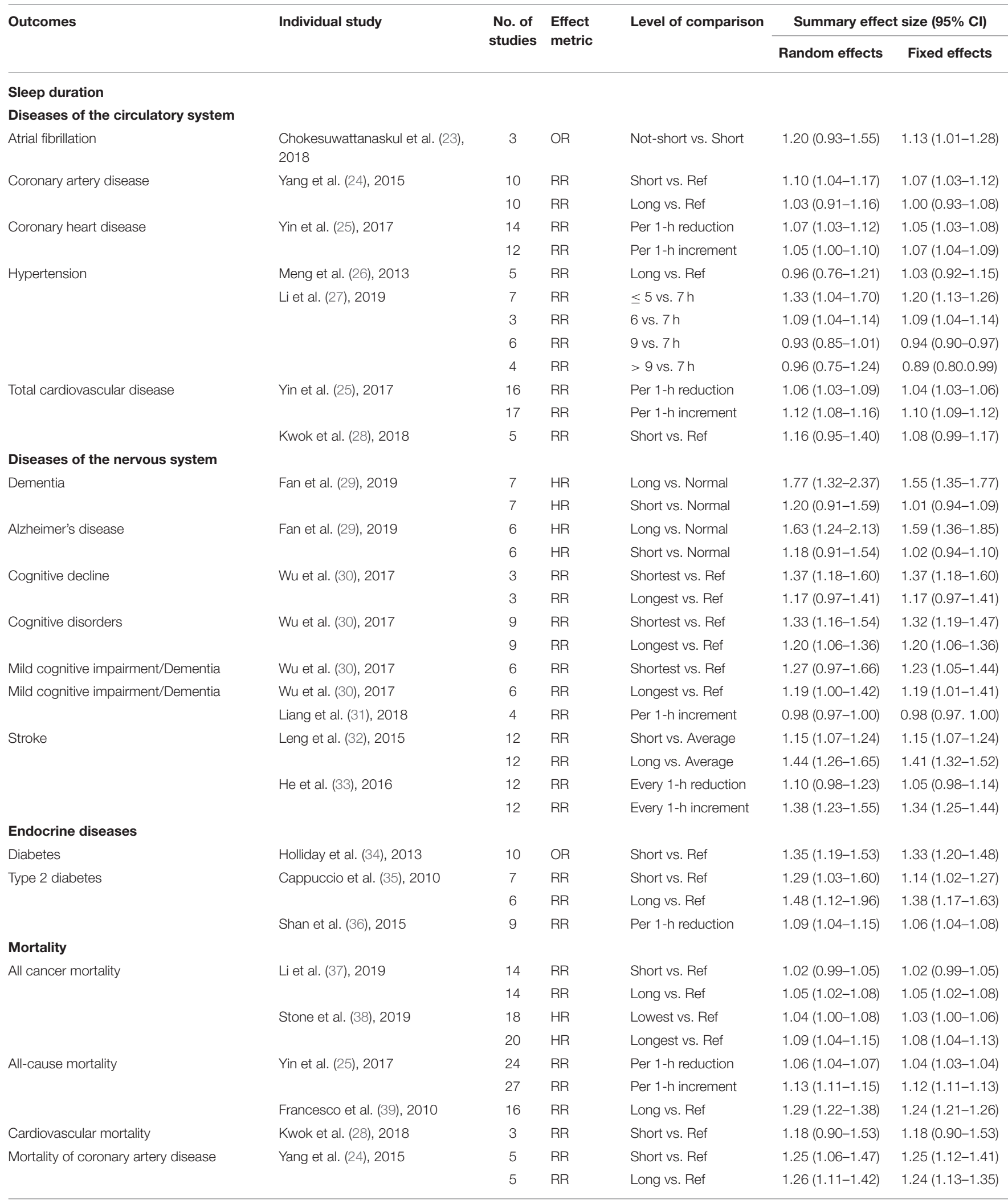


TABLE 1 | Continued

\begin{tabular}{|c|c|c|c|c|c|c|}
\hline \multirow[t]{2}{*}{ Outcomes } & \multirow[t]{2}{*}{ Individual study } & \multirow{2}{*}{$\begin{array}{l}\text { No. of } \\
\text { studies }\end{array}$} & \multirow{2}{*}{$\begin{array}{l}\text { Effect } \\
\text { metric }\end{array}$} & \multirow[t]{2}{*}{ Level of comparison } & \multicolumn{2}{|c|}{ Summary effect size $(95 \% \mathrm{CI})$} \\
\hline & & & & & Random effects & Fixed effects \\
\hline \multirow[t]{4}{*}{ Mortality of coronary heart disease } & Kwok et al. (28), 2018 & 3 & $\mathrm{RR}$ & Short vs. Ref & $1.44(0.74-2.83)$ & $1.51(1.05-2.16)$ \\
\hline & Kwok et al. (28), 2018 & 5 & $\mathrm{RR}$ & Short vs. Ref & $1.29(1.10-1.51)$ & $1.29(1.17-1.43)$ \\
\hline & Kwok et al. (28), 2018 & 4 & $\mathrm{RR}$ & Short vs. Ref & $1.12(0.98-1.27)$ & $1.12(1.01-1.24)$ \\
\hline & Kwok et al. (28), 2018 & 6 & $\mathrm{RR}$ & Long vs. Ref & $1.36(1.17-1.59)$ & $1.39(1.26-1.53)$ \\
\hline Mortality of coronary heart disease & Kwok et al. (28), 2018 & 3 & $\mathrm{RR}$ & Long vs. Ref & $1.24(1.00-1.53)$ & $1.24(1.00-1.53)$ \\
\hline $\begin{array}{l}\text { Mortality (all-cause and } \\
\text { cause-specific) }\end{array}$ & Gallicchio et al. (40), 2009 & 16 & $\mathrm{RR}$ & Short vs. Ref & $1.10(1.06-1.15)$ & $1.10(1.08-1.12)$ \\
\hline \multirow[t]{2}{*}{ Prostate cancer mortality } & Liu et al. (41), 2020 & 6 & $\mathrm{RR}$ & Short vs. Ref & $0.99(0.91-1.07)$ & $0.99(0.91-1.07)$ \\
\hline & & 6 & $\mathrm{RR}$ & Long vs. Ref & $0.88(0.75-1.04)$ & $0.94(0.87-1.02)$ \\
\hline \multirow[t]{2}{*}{ Stroke mortality } & Li et al. (42), 2016 & 4 & $\mathrm{RR}$ & Per 1-h reduction & 1.05 (0.99-1.11) & $1.05(0.99-1.11)$ \\
\hline & & 11 & $\mathrm{RR}$ & Per 1-h increment & $1.17(1.13-1.20)$ & $1.17(1.13-1.20)$ \\
\hline \multicolumn{7}{|l|}{ Neoplasms } \\
\hline \multirow[t]{2}{*}{ Breast cancer } & Wong et al. (43), 2020 & 15 & $\mathrm{RR}$ & Short vs. Ref & $0.99(0.97-1.01)$ & $0.99(0.98-1.01)$ \\
\hline & & 15 & $\mathrm{RR}$ & Long vs. Ref & $1.00(0.96-1.04)$ & $1.01(0.98-1.04)$ \\
\hline \multirow[t]{4}{*}{ Cancer } & Lu et al. (44), 2013 & 9 & $\mathrm{RR}$ & Short vs. Ref & $1.05(0.90-1.24)$ & $1.02(0.93-1.11)$ \\
\hline & & 9 & $\mathrm{RR}$ & Long vs. Ref & $0.92(0.76-1.12)$ & $0.97(0.88-1.06)$ \\
\hline & Zhao et al. (45), 2013 & 9 & $\mathrm{HR}$ & Per 1-h reduction & 1.06 (0.92-1.22) & $1.01(0.94-1.09)$ \\
\hline & & 12 & $\mathrm{HR}$ & Per 1-h increment & $0.91(0.78-1.07)$ & $0.98(0.91-1.06)$ \\
\hline \multicolumn{7}{|l|}{ Nutritional diseases } \\
\hline \multirow[t]{2}{*}{ Obesity } & Miller et al. (46), 2020 & 13 & $\mathrm{RR}$ & Per an additional hour & $1.54(1.33-1.77)$ & $1.51(1.43-1.60)$ \\
\hline & Wu et al. (47), 2015 & 13 & OR & Short vs. Ref & $1.71(1.36-2.14)$ & $2.18(2.13-2.22)$ \\
\hline Overweight & Ruan et al. (48), 2015 & 7 & OR & Lowest vs. Highest & 1.79 (1.39-2.31) & $2.22(2.17-2.26)$ \\
\hline Overweight and obesity & Fatima et al. (49), 2015 & 11 & OR & Short vs. Ref & 1.56 (1.24-1.98) & $2.20(2.16-2.25)$ \\
\hline \multirow[t]{2}{*}{ Overweight or obesity } & Miller et al. (50), 2018 & 7 & $\mathrm{RR}$ & Highest vs. Lowest & $1.40(1.18-1.65)$ & $1.28(1.16-1.41)$ \\
\hline & & 8 & $\mathrm{RR}$ & Highest vs. Lowest & $1.57(1.40-1.76)$ & $1.61(1.51-1.72)$ \\
\hline \multicolumn{7}{|l|}{ Other outcomes } \\
\hline \multirow[t]{2}{*}{ Depression } & Zhai et al. (51), 2015 & 6 & $\mathrm{RR}$ & Short vs. Ref & $1.30(1.04-1.64)$ & $1.30(1.04-1.64)$ \\
\hline & & 4 & $\mathrm{RR}$ & Long vs. Ref & $1.41(1.04-1.92)$ & $1.41(1.04-1.92)$ \\
\hline \multirow[t]{2}{*}{ Dyslipidaemia } & Kruisbrink et al. (52), 2017 & 6 & $\mathrm{RR}$ & Short vs. Ref & $1.01(0.92-1.11)$ & $1.05(0.99-1.10)$ \\
\hline & & 6 & $R R$ & Long vs. Ref & $0.98(0.87-1.10)$ & $0.94(0.88-1.00)$ \\
\hline Gestational diabetes mellitus & Zhang et al. (53), 2020 & 4 & $\mathrm{RR}$ & Long vs. Normal & $1.19(1.05-1.35)$ & $1.19(1.05-1.35)$ \\
\hline \multirow[t]{3}{*}{ Gestational diabetes mellitus } & Zhang et al. (53), 2020 & 4 & $\mathrm{RR}$ & Short vs. Not-short & $2.02(1.31-3.11)$ & $2.02(1.31-3.11)$ \\
\hline & Xu et al. (54), 2018 & 5 & OR & Short vs. Ref & $1.58(0.99-2.52)$ & $1.37(1.05-1.80)$ \\
\hline & & 3 & OR & Long vs. Ref & $1.28(1.10-1.49)$ & $1.28(1.10-1.49)$ \\
\hline \multirow{2}{*}{$\begin{array}{l}\text { The developing or dying of coronary heart } \\
\text { disease }\end{array}$} & Cappuccio et al. (55), 2011 & 7 & $\mathrm{RR}$ & Short vs. Ref & $1.48(1.22-1.80)$ & $1.48(1.31-1.68)$ \\
\hline & & 7 & $\mathrm{RR}$ & Long vs. Ref & $1.38(1.15-1.66)$ & $1.41(1.26-1.59)$ \\
\hline \multirow[t]{2}{*}{ The developing or dying of stroke } & Cappuccio et al. (55), 2011 & 4 & $\mathrm{RR}$ & Short vs. Ref & $1.15(1.00-1.32)$ & $1.15(1.00-1.32)$ \\
\hline & & 4 & $\mathrm{RR}$ & Long vs. Ref & $1.65(1.45-1.87)$ & $1.65(1.45-1.87)$ \\
\hline The developing or dying of total & Cappuccio et al. (55), 2011 & 7 & $R R$ & Short vs. Ref & $1.03(0.93-1.15)$ & $1.03(0.93-1.15)$ \\
\hline & & 8 & $\mathrm{RR}$ & Long vs. Ref & $1.41(1.20-1.67)$ & $1.42(1.30-1.54)$ \\
\hline Sleep quality & & & & & & \\
\hline All-cause mortality & Kwok et al. (28), 2018 & 10 & $\mathrm{RR}$ & Poor vs. Good & $1.03(0.93-1.14)$ & $1.03(0.98-1.09)$ \\
\hline Cardiovascular mortality & Kwok et al. (28), 2018 & 4 & $\mathrm{RR}$ & Poor vs. Good & $0.96(0.82-1.13)$ & $0.96(0.82-1.13)$ \\
\hline Coronary heart disease & Kwok et al. (28), 2018 & 4 & $\mathrm{RR}$ & Poor vs. Good & $1.44(1.09-1.90)$ & $1.32(1.12-1.56)$ \\
\hline Diabetes mellitus & Anothaisintawee et al. (56), 2015 & 11 & $\mathrm{RR}$ & Poor vs. Not- Poor & $1.40(1.21-1.63)$ & $1.32(1.28-1.36)$ \\
\hline Gestational diabetes mellitus & Zhang et al. (53), 2020 & 4 & $\mathrm{RR}$ & Poor vs. Not- Poor & $1.27(1.11-1.44)$ & $1.27(1.11-1.44)$ \\
\hline Inflammatory bowel disease & Hao et al. (57), 2020 & 3 & OR & Poor vs. Not- Poor & $2.54(1.37-4.71)$ & $2.38(1.71-3.31)$ \\
\hline Preterm birth & Wang et al. (58), 2020 & 5 & $\mathrm{RR}$ & Poor vs. Good & $1.54(1.18-2.00)$ & $1.26(1.15-1.38)$ \\
\hline
\end{tabular}

$\mathrm{Cl}$, confidence interval; OR, odds ratio; $R R$, relative risk; HR: hazard ratio.

All statistical tests were two-sided. 
TABLE 2 | Level of evidence for the association of sleep duration/quality for health outcomes.

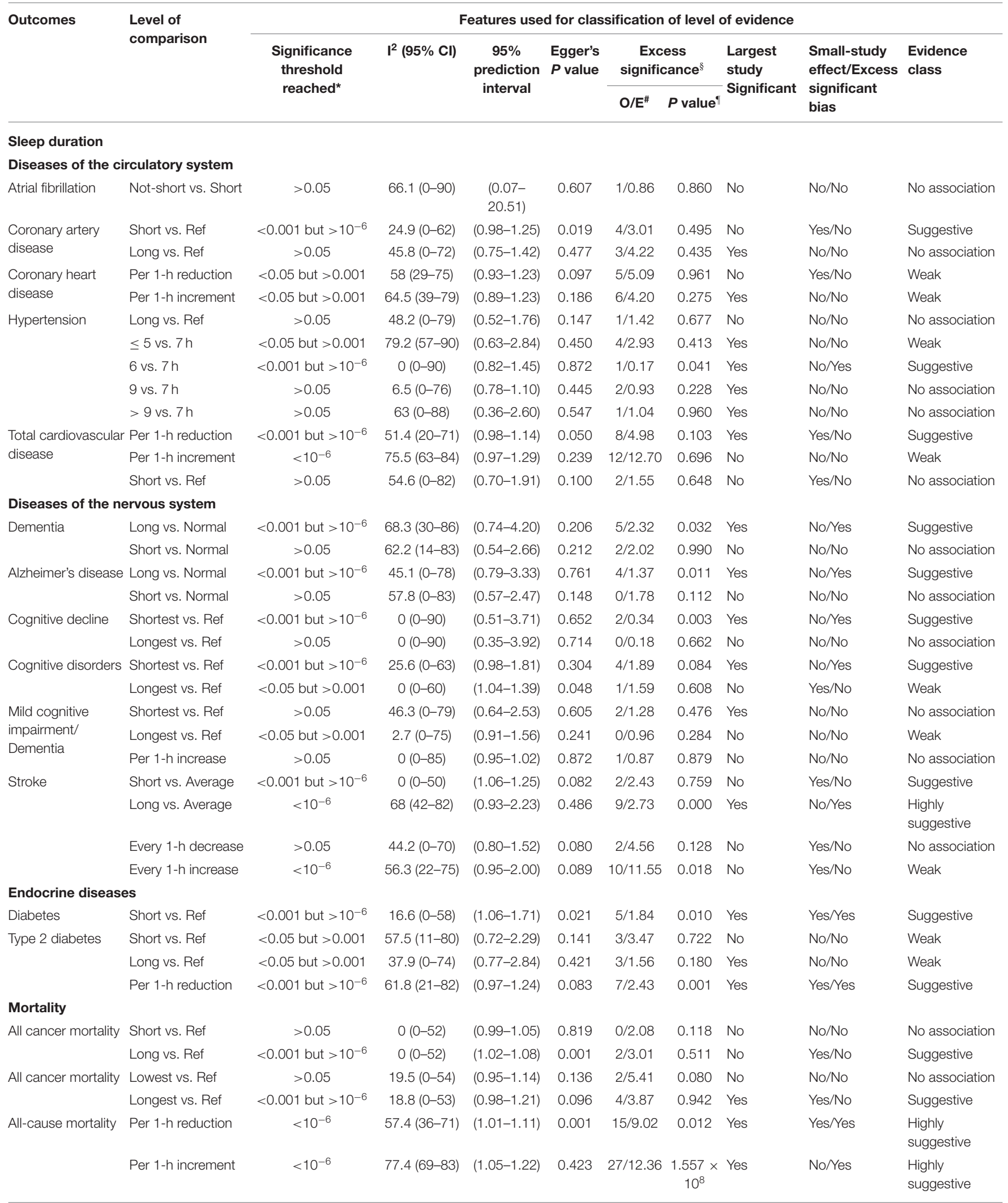


TABLE 2 | Continued

\begin{tabular}{|c|c|c|c|c|c|c|c|c|c|c|}
\hline \multirow[t]{2}{*}{ Outcomes } & \multirow{2}{*}{$\begin{array}{l}\text { Level of } \\
\text { comparison }\end{array}$} & \multicolumn{9}{|c|}{ Features used for classification of level of evidence } \\
\hline & & $\begin{array}{c}\text { Significance } \\
\text { threshold } \\
\text { reached }^{\star}\end{array}$ & $\mathrm{I}^{2}(95 \% \mathrm{Cl})$ & $\begin{array}{c}95 \% \\
\text { prediction } \\
\text { interval }\end{array}$ & $\begin{array}{l}\text { Egger's } \\
P \text { value }\end{array}$ & \multicolumn{2}{|c|}{$\begin{array}{c}\text { Excess } \\
\text { significance }^{\S}\end{array}$} & $\begin{array}{l}\text { Largest } \\
\text { study } \\
\text { Significant }\end{array}$ & $\begin{array}{l}\text { Small-study } \\
\text { effect/Excess } \\
\text { significant } \\
\text { bias }\end{array}$ & $\begin{array}{l}\text { Evidence } \\
\text { class }\end{array}$ \\
\hline & Long vs. Ref & $<10^{-6}$ & $71(57-80)$ & $(1.03-1.62)$ & 0.160 & $15 / 8.96$ & 0.002 & Yes & No/Yes & $\begin{array}{l}\text { Highly } \\
\text { suggestive }\end{array}$ \\
\hline \multirow{2}{*}{$\begin{array}{l}\text { Mortality of } \\
\text { coronary artery } \\
\text { disease }\end{array}$} & Short vs. Ref & $<0.05$ but $>0.001$ & $42.6(0-75)$ & $(0.82-1.90)$ & 0.644 & $4 / 1.88$ & 0.050 & Yes & No/Yes & Weak \\
\hline & Long vs. Ref & $<0.001$ but $>10^{-6}$ & $38.9(0-73)$ & $(0.93-1.70)$ & 0.493 & $3 / 3.13$ & 0.901 & No & No/No & Suggestive \\
\hline $\begin{array}{l}\text { Mortality of } \\
\text { coronary heart } \\
\text { disease }\end{array}$ & Short vs. Ref & $>0.05$ & $55.5(0-87)$ & $\begin{array}{c}(0.00- \\
1,535.63)\end{array}$ & 0.667 & $1 / 0.73$ & 0.569 & No & No/No & No association \\
\hline $\begin{array}{l}\text { Mortality (all-cause } \\
\text { and cause-specific) }\end{array}$ & Short vs. Ref & $<0.001$ but $>10^{-6}$ & $20.5(0-56)$ & $(1.01-1.21)$ & 0.712 & $4 / 2.95$ & 0.500 & Yes & No/No & Suggestive \\
\hline \multirow{2}{*}{$\begin{array}{l}\text { Prostate cancer } \\
\text { mortality }\end{array}$} & Short vs. Ref & $>0.05$ & $0(0-75)$ & $(0.88-1.10)$ & 0.466 & 0/0.86 & 0.316 & No & No/No & No association \\
\hline & Long vs. Ref & $>0.05$ & $55.6(0-82)$ & $(0.56-1.40)$ & 0.241 & $2 / 1.52$ & 0.651 & No & $\mathrm{No} / \mathrm{No}$ & No association \\
\hline \multirow[t]{2}{*}{ Stroke mortality } & Per 1-h reduction & $>0.05$ & $0(0-71)$ & $(0.98-1.12)$ & 0.996 & $1 / 1.27$ & 0.774 & No & No/No & No association \\
\hline & Per 1-h increase & $<10^{-6}$ & $1.5(0-61)$ & $(1.12-1.21)$ & 0.490 & $8 / 1.92$ & $\begin{array}{c}1.001 \times \\
10^{7}\end{array}$ & Yes & No/Yes & $\begin{array}{l}\text { Highly } \\
\text { suggestive }\end{array}$ \\
\hline \multicolumn{11}{|l|}{ Neoplasms } \\
\hline \multirow[t]{2}{*}{ Breast cancer } & Short vs. Ref & $>0.05$ & $7.5(0-44)$ & $(0.96-1.03)$ & 0.347 & $2 / 2.57$ & 0.695 & No & No/No & No association \\
\hline & Long vs. Ref & $>0.05$ & $11.2(0-49)$ & $(0.93-1.07)$ & 0.065 & 0/2.69 & 0.070 & No & Yes/No & No association \\
\hline Overweight & Lowest vs. Highest & $<0.001$ but $>10^{-6}$ & 76.5 (53-88) & $(0.85-3.78)$ & 0.167 & $7 / 3.09$ & 0.003 & Yes & No/Yes & Suggestive \\
\hline $\begin{array}{l}\text { Overweight and } \\
\text { obesity }\end{array}$ & Short vs. Ref & $<0.001$ but $>10^{-6}$ & $87.1(80-92)$ & (0.69-3.56) & 0.026 & $10 / 5.75$ & 0.010 & Yes & Yes/Yes & Suggestive \\
\hline \multirow{2}{*}{$\begin{array}{l}\text { Overweight or } \\
\text { obesity }\end{array}$} & Highest vs. Lowest & $<0.001$ but $>10^{-6}$ & $40.5(0-75)$ & (0.94-2.09) & 0.002 & $5 / 2.00$ & 0.012 & Yes & Yes/Yes & Suggestive \\
\hline & Highest vs. Lowest & $<10^{-6}$ & $22.7(0-64)$ & $(1.23-2.00)$ & 0.521 & $6 / 1.49$ & $\begin{array}{c}4.063 \times \\
10^{5}\end{array}$ & Yes & No/Yes & $\begin{array}{l}\text { Highly } \\
\text { suggestive }\end{array}$ \\
\hline \multicolumn{11}{|l|}{ Other outcome } \\
\hline \multirow[t]{2}{*}{ Depression } & Short vs. Ref & $<0.05$ but $>0.001$ & $0(0-71)$ & $(0.97-1.76)$ & 0.965 & $1 / 1.09$ & 0.925 & No & No/No & Weak \\
\hline & Long vs. Ref & $<0.05$ but $>0.001$ & $0(0-79)$ & (0.86-2.32) & 0.526 & $0 / 0.77$ & 0.328 & No & No/No & Weak \\
\hline Dyslipidaemia & Short vs. Ref & $>0.05$ & $62.2(29-80)$ & $(0.75-1.36)$ & 0.147 & $4 / 4.57$ & 0.585 & Yes & No/No & No association \\
\hline & Long vs. Ref & $>0.05$ & 63.9 (38-79) & $(0.65-1.48)$ & 0.249 & $3 / 5.79$ & $\begin{array}{c}6.291 \times \\
10^{10}\end{array}$ & No & No/No & No association \\
\hline Gestational & Long vs. Normal & $<0.05$ but $>0.001$ & $0(0-85)$ & $(0.90-1.58)$ & 0.883 & $1 / 0.67$ & 0.657 & Yes & No/No & Weak \\
\hline diabetes mellitus & Short vs. Not-short & $<0.001$ but $>10^{-6}$ & $0(0-85)$ & $(0.78-5.19)$ & 0.003 & $1 / 0.58$ & 0.545 & No & Yes/No & Weak \\
\hline & Short vs. Ref & $>0.05$ & $57.1(0-83)$ & $(0.42-5.93)$ & 0.031 & $3 / 1.90$ & 0.312 & No & Yes/No & No association \\
\hline & Long vs. Ref & $<0.001$ but $>10^{-6}$ & $0(0-85)$ & $(0.92-1.78)$ & 0.771 & $1 / 0.36$ & 0.260 & Yes & No/No & Suggestive \\
\hline The developing or & Short vs. Ref & $<0.001$ but $>10^{-6}$ & $43.3(0-72)$ & $(0.91-2.41)$ & 0.962 & $6 / 3.23$ & 0.036 & Yes & No/Yes & Suggestive \\
\hline $\begin{array}{l}\text { dying of coronary } \\
\text { heart disease }\end{array}$ & Long vs. Ref & $<0.001$ but $>10^{-6}$ & $49(1-74)$ & (0.84-2.29) & 0.913 & $5 / 4.96$ & 0.976 & Yes & No/No & Suggestive \\
\hline
\end{tabular}


TABLE 2 | Continued

\begin{tabular}{|c|c|c|c|c|c|c|c|c|c|c|}
\hline \multirow[t]{3}{*}{ Outcomes } & \multirow{3}{*}{$\begin{array}{l}\text { Level of } \\
\text { comparison }\end{array}$} & \multicolumn{9}{|c|}{ Features used for classification of level of evidence } \\
\hline & & \multirow{2}{*}{$\begin{array}{c}\text { Significance } \\
\text { threshold } \\
\text { reached }^{\star}\end{array}$} & \multirow[t]{2}{*}{$\mathrm{I}^{2}(95 \% \mathrm{Cl})$} & \multirow{2}{*}{$\begin{array}{c}95 \% \\
\text { prediction } \\
\text { interval }\end{array}$} & \multirow{2}{*}{$\begin{array}{l}\text { Egger's } \\
P \text { value }\end{array}$} & \multicolumn{2}{|c|}{$\begin{array}{c}\text { Excess } \\
\text { significance }^{\S}\end{array}$} & \multirow{2}{*}{$\begin{array}{l}\text { Largest } \\
\text { study } \\
\text { Significant }\end{array}$} & \multirow{2}{*}{$\begin{array}{l}\text { Small-study } \\
\text { effect/Excess } \\
\text { significant } \\
\text { bias }\end{array}$} & \multirow{2}{*}{$\begin{array}{l}\text { Evidence } \\
\text { class }\end{array}$} \\
\hline & & & & & & O/E\# & $P$ value ${ }^{\text {Il }}$ & & & \\
\hline \multirow{2}{*}{$\begin{array}{l}\text { The developing or } \\
\text { dying of stroke }\end{array}$} & Short vs. Ref & $<0.05$ but $>0.001$ & $0(0-75)$ & $(0.95-1.39)$ & 0.304 & $0 / 0.82$ & 0.310 & No & $\mathrm{No} / \mathrm{No}$ & Weak \\
\hline & Long vs. Ref & $<10^{-6}$ & $0(0-75)$ & $(1.38-1.97)$ & 0.955 & $4 / 0.47$ & $\begin{array}{c}4.725 \times \\
10^{8}\end{array}$ & Yes & No/Yes & $\begin{array}{l}\text { Highly } \\
\text { suggestive }\end{array}$ \\
\hline \multirow{2}{*}{$\begin{array}{l}\text { The developing or } \\
\text { dying of total } \\
\text { cardiovascular } \\
\text { disease }\end{array}$} & Short vs. Ref & $>0.05$ & $0(0-60)$ & $(0.92-1.16)$ & 0.470 & $0 / 1.01$ & 0.279 & No & No/No & No association \\
\hline & Long vs. Ref & $<0.001$ but $>10^{-6}$ & $59.8(26-78)$ & $(0.86-2.31)$ & 0.794 & $6 / 2.89$ & 0.022 & Yes & No/Yes & Suggestive \\
\hline \multicolumn{11}{|l|}{ Sleep quality } \\
\hline All-cause mortality & Poor vs. Good & $>0.05$ & $0.6(14-79)$ & $(0.79-1.35)$ & 0.917 & $3 / 2.89$ & 1.000 & Yes & No/No & No association \\
\hline $\begin{array}{l}\text { Cardiovascular } \\
\text { mortality }\end{array}$ & Poor vs. Good & $>0.05$ & $0(0-85)$ & $(0.67-1.37)$ & 0.303 & $0 / 0.31$ & 1.000 & No & No/No & No association \\
\hline $\begin{array}{l}\text { Coronary heart } \\
\text { disease }\end{array}$ & Poor vs. Good & $<0.05$ but $>0.001$ & $53.2(0-85)$ & $(0.51-4.10)$ & 0.075 & $1 / 1.08$ & 1.000 & No & Yes/No & Weak \\
\hline Diabetes mellitus & Poor vs. Not- Poor & $<0.001$ but $>10^{-6}$ & $84.1(73-91)$ & $(0.89-2.22)$ & 0.679 & 8/4.04 & 0.013 & Yes & No/Yes & Suggestive \\
\hline $\begin{array}{l}\text { Gestational } \\
\text { diabetes mellitus }\end{array}$ & Poor vs. Not- Poor & $<0.001$ but $>10^{-6}$ & $0(0-85)$ & $(0.95-1.68)$ & 0.807 & $2 / 0.50$ & 0.024 & Yes & No/Yes & Suggestive \\
\hline $\begin{array}{l}\text { Inflammatory bowel } \\
\text { disease }\end{array}$ & Poor vs. Not- Poor & $<0.05$ but $>0.001$ & $62.3(0-89)$ & $\begin{array}{c}(0- \\
1,940.14)\end{array}$ & 0.683 & $2 / 0.87$ & 0.148 & Yes & No/No & Weak \\
\hline Preterm birth & Poor vs. Good & $<0.001$ but $>10^{-6}$ & 76.7 (43-90) & $(0.67-3.51)$ & 0.039 & $5 / 1.73$ & 0.002 & Yes & Yes/Yes & Weak \\
\hline
\end{tabular}

Cl, confidence interval.

$\S$ Expected number of statistically significant studies using the point estimate of the largest study (smallest standard error) as the plausible effect size.

${ }^{\star} P$ value under the random-effects model.

\# Observed/Expected number of statistically significant studies.

IP value of the excess statistical significance test.

All statistical tests two sided.

\section{Small-Study Effects and Excess Significance Bias}

According to Egger's test, evidence of small-study effects was observed in $21(27 \%)$ of 78 meta-analyses and $2(29 \%)$ of 7 meta-analyses about duration and quality of sleep, respectively (Table 2). When taking the largest study estimate as to the plausible effect size, 22 (28\%) of 78 meta-analyses and $3(43 \%)$ of 7 meta-analyses about duration and quality of sleep respectively showed evidence of excess significance (Table 2).

\section{Methodological Quality of the Meta-Analyses}

The methodological quality of the included studies regarding sleep duration $(\mathrm{n}=33)$ and sleep quality $(\mathrm{n}=5)$ was assessed by AMSTAR-1, which contained 11 items for scoring. Figure 2 provides a breakdown of AMSTAR-1 levels for studies representing each study. For sleep duration, the median AMSTAR-1 score achieved across all studies was 6 out of 11 (range from 2 to 9). The studies were rated at three levels: 15\% were rated as "high," 79\% were rated as "moderate," and 6\% were classified as "low." For sleep quality, the median AMSTAR1 score achieved across all studies was 7 (range from 5 to 8). Approximately $40 \%$ were rated as being of "high," $60 \%$ as "moderate" quality, and no meta-analysis was categorized into low quality according to the AMSTAR-1 criteria. The common flaws were that gray literature was not considered in the literature search (item 4), and the list of excluded studies was not presented (item 5).

\section{Evidence Grading}

For sleep duration, no association presented convincing evidence, the only evidence of long sleep duration with an increased risk of all-cause mortality was categorized as highly suggestive, and the methodological quality was moderate (Table 2). Moreover, suggestive evidence supported the associations between long sleep and increased risk of 5 health outcomes (stroke, dyslipidaemia, mortality of coronary heart disease, stroke mortality, and the development or death of stroke); short sleep duration and increased risk of overweight and/or obesity. Moreover, 14 associations were supported by weak evidence. The remaining 31 associations were not confirmed. The detailed results of the analyses on which the evidence ratings were based are shown in Table 2.

For sleep quality, no association presented convincing or highly suggestive evidence, whereas suggestive evidence suggested that poor sleep quality was associated with an increased risk of diabetes mellitus and gestational diabetes mellitus. Moreover, 3 associations were supported by weak evidence and 2 associations were not confirmed. 


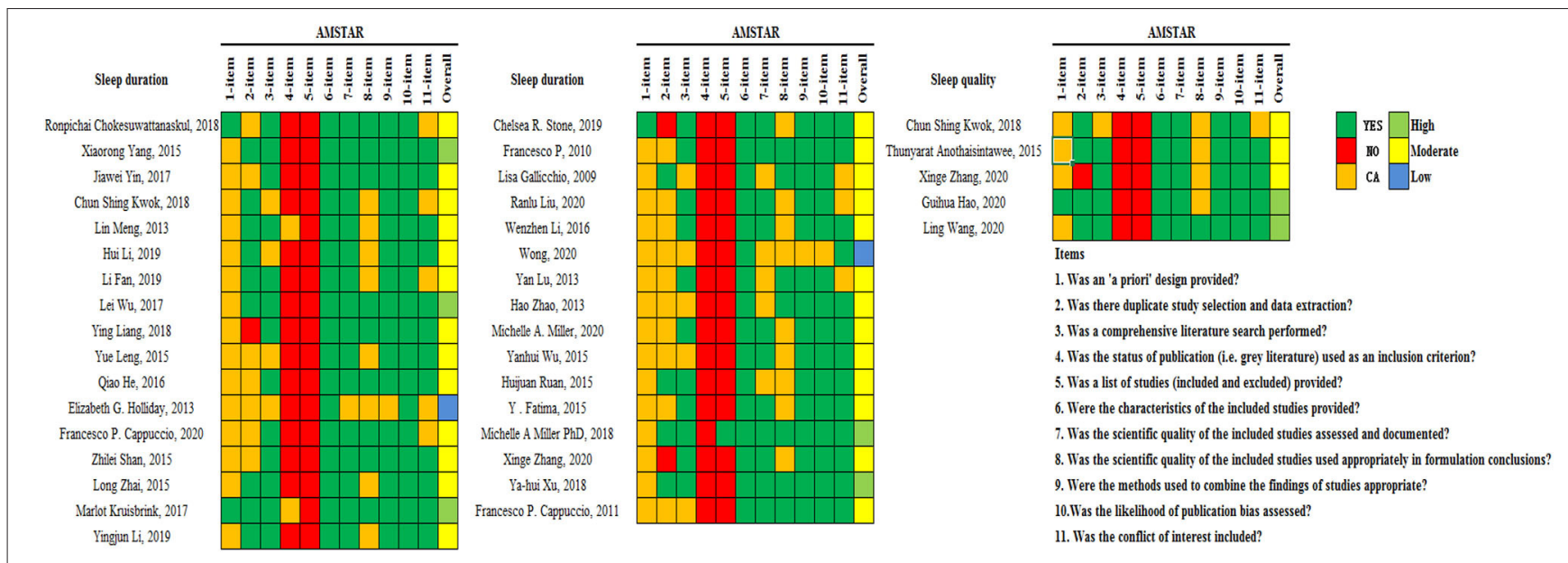

FIGURE 2 | Detailed evaluation of the methodological quality with AMSTAR-1.

\section{DISCUSSION}

In this umbrella review, to objectively assess the strength of associations between duration and quality of sleep and health outcomes, we performed a comprehensive overview by incorporating evidence from the current systematic reviews and meta-analyses of prospective studies. Overall, 85 published meta-analyses were included, and $52(61 \%)$ were nominally statistically significant at $P<0.05$ under the random-effects models. Although the study confirmed that short/long sleep duration or poor sleep quality was associated with an increase in the important health outcomes, the mechanisms do not seem straightforward.

In this umbrella review, evidence of the association of long sleep duration with an increased risk of all-cause mortality (25), was the only one categorized as highly suggestive, and the methodological quality was moderate in the above outcome. An association between long sleep and an increased risk of allcause mortality was reported previously in studies with high quality and large sample sizes (59-62), which was consistent with our results. Heslop and colleagues (63), however, analyzed data from a workplace-based study of Scottish men and women who were followed over a 25 -year period and found that long sleep was associated with decreased risk of all-cause mortality in men. However, this study reported RRs with only 3 quantitative categories of sleep duration. Meanwhile, long sleep duration was defined as $>8 \mathrm{~h}$, which may result in inaccurate evaluation of extremely long sleep. To date, no published studies have demonstrated a possible mechanism mediating the effect of long sleep as a cause of mortality. The association between a long duration of sleep and mortality may be explained by residual confounding and comorbidities (64). In particular, depressive symptoms, low socioeconomic status, low level of physical activity, unemployment, undiagnosed health conditions, poor general health, and cancer-related fatigue have all been shown to be associated with long sleep (64).

Suggestive evidence has shown that long sleep duration is positively linked with the morbidity of stroke (32) and mortality of stroke per 1-h increase in sleep duration (42). At present, the biological mechanisms of the relationship between long sleep and stroke are not clear. One important biological pathway is inflammation, as long sleep periods have been associated with an increased level of inflammatory biomarkers, such as C-reactive protein and interleukin-6 (65-68). Interestingly, a number of studies have associated long sleep with cardiovascular conditions including atrial fibrillation, carotid artery atherosclerosis, and left ventricular mass, which might have predisposed one to the risk of stroke (69-73). Meanwhile, some studies suggested an association for long sleep and stroke only among those with limited physical function (74) or with a history of hypertension (75). Another possible biological pathway is due to sleep disorders such as sleep-disordered breathing (76). Decreased cerebral blood flow and raised intracranial pressure occurred during apneic events in some studies $(77,78)$, and cerebral hypoperfusion may also occur during wakefulness in sleep apnea patients (79). Klingelhofer et al. (80) found that blood flow in the middle cerebral artery during apneic showed rapid increases and decreases in velocity. Such changes could incline vulnerable individuals to ischemic or hemorrhagic events (76). Several epidemiological studies have explored this association. A previous meta-analysis indicated that long sleep duration was associated with an increased risk of stroke (55), but they did not use a dose-response analysis to determine the association (55). A meta-analysis by Ge showed a significantly increased risk of stroke incidence and mortality at long sleep durations in both cohort and cross-sectional studies. Their subgroup analysis also showed that long sleep duration was a statistical stroke risk in both sexes and in Asians (81). Those results were in accordance with ours. However, the relationship between sleep duration and stroke may be related to stroke types (82), age (83), gender and race (84), and high-quality studies are therefore needed to explore this matter.

We also found suggestive evidence that long sleep duration is associated with an increased risk of mortality of coronary heart disease (28). Khan et al. (85) found that there is a significant association of coronary heart disease in the top 
quartile of sleep duration compared to those in the bottom quartile. Those results were in accordance with ours. However, further adjustment for risk factors including systolic blood pressure, history of cardiovascular disease, diabetes, smoking, alcohol use, renal function and serum Low-Density Lipoprotein cholesterol attenuated the associations with fatal coronary heart disease. However, the average sleep duration in Khan's study was $9.1 \mathrm{~h}$ with the lowest quartile being $8.2 \mathrm{~h}$. This is longer than previously reported data from Western populations. Long sleep duration has been related to systemic inflammation, an increase in cytokines and changes in several metabolic pathways (68). The lack of physiological challenge due to increased sleep has also been proposed as a mechanism that may increase mortality (86). Longer sleep duration has also been linked to depression and other psychiatric disorders, which are known to be associated with increased cardiovascular disease events $(51,87)$. However, all these proposed mechanisms are speculative at best and require more research.

Suggestive evidence also showed that short sleep duration is linked with the increased risk of overweight or obesity only observed in children (50). There are several lines of evidence to suggest plausible mechanisms. Sleep deprivation is associated with various hormonal responses that may affect both hunger and satiety, leading to appetite dysregulation. These include lower leptin and higher ghrelin levels $(88,89)$, which would increase appetite. Sleep deprivation has effects on endocannabinoids which regulate a variety of central nervous system processes including appetite (90). Changes in factors that affect metabolism, including insulin and glucose metabolism, cortisol, growth hormone and thyroid stimulating hormone are also important $(48,91-95)$. In turn, obesity predisposes individuals to metabolic dysfunction that can cause sleep apnea, which leads to short sleep duration (96). Activation of inflammatory pathways by short sleep periods may be implicated in the development of obesity (97) and it can up and downregulate the expression of genes involved in oxidative stress and metabolism (98). Finally, insufficient sleep is associated with alterations in attention, impulse control, mood, motivation, and judgment, and all of these factors could potentially influence eating behaviors, energy intake, and ultimately BMI in children (99).

Regarding sleep quality, we found that poor sleep quality is associated with an increased risk of diabetes mellitus (56) and gestational diabetes mellitus (53). This result is consistent with the results of a previous meta-analysis, which concluded that sleep quality may be a novel and independent risk factor for poorer glycemic control in type 2 diabetes patients (100). Poor sleep quality as defined by the presence of one or more insomnia symptoms included in the Diagnostic and Statistical Manual of Mental Disorders diagnostic criteria was associated with a $40 \%$ increase in the risk of developing diabetes. Poor self-reported sleep quality may also be linked with other comorbid conditions, such as depression, undiagnosed obstructive sleep apnea and sleep deprivation, which are risk factors for diabetes. Adjusting for these covariates still resulted in a significant association between poor sleep quality and incident diabetes in several studies $(101,102)$. Pregnant women with poor sleep quality had an increased risk of gestational diabetes mellitus (GDM). In addition to its direct effect on GDM, sleep quality has been considered a moderator of the association between sleep duration and GDM risk (103). The potential pathophysiological mechanisms between poor sleep quality and glucose intolerance have been well established, including decreased brain glucose utilization (104106), sympathetic nervous system overactivity (107, 108), alterations in the hypothalamic-pituitary-adrenal axis and growth hormone (109-111), elevated systemic inflammatory response $(112,113)$, reduction in the percentage of slow wave sleep (114), adipocyte dysfunction (115), changes in appetiteregulating hormones (88), and increased obesity risk (95).

To our knowledge, the present study is the first umbrella review to quantitatively evaluate the existing evidence of the associations between duration and quality of sleep and health outcomes. The main strength of our umbrella review was to provide a comprehensive summary and evaluation of the credibility and validity of evidence of duration and quality of sleep and health outcomes according to the assessment results of a series of statistical analyses. In addition, we searched three databases through a rigorous strategy, and two authors independently extracted the information. Moreover, we followed the AMSTAR-1 criteria to assess the methodological quality of selected studies in our umbrella review, and most of the investigated meta-analyses achieved a moderate-to-high quality score. We used standardized criteria to explore the extent of heterogeneity and potential bias among the included studies and further assessed the strength of claimed associations to identify which was the most credible evidence. We also used the criteria of evidence grading to evaluate the evidence categorization.

Nevertheless, several limitations should be noted when interpreting the results. Firstly, we failed to find convincing evidence for the relation of sleep and health outcomes. In addition, the quality of the evidence was rated weak or not confirmed for $39 \%$ of the associations $(n=33)$. Thus, further research is needed for outcomes for which the certainty of evidence was rated weak or not confirmed. Secondly, owing to the limited studies, we failed to conduct subgroup analysis (e.g., exploring by age, sex, geographical location), or sensitive analysis (e.g., excluding studies with high risks), and other relevant factors might have been missed. Many meta-analyses lacked doseresponse information and compared high vs. low sleep duration without defining thresholds for these categories. Thirdly, we only evaluated published meta-analyses of prospective studies with available data, therefore, meta-analyses of randomized controlled trials were not included in our study. Fourthly, for sleep quality, only systematic reviews and meta-analyses assessing sleep quality by the PSQI questionnaire were included in this umbrella review. The PSQI is currently the only standardized clinical instrument that covers a broad range of indicators relevant to sleep quality (116). Lastly, we did not examine any error of the meta-analyses or the quality of the primary studies, as these were beyond the scope of our umbrella review. Our findings appear to be very convincing, but one may need to practice caution in terms of considering the implications of the results in the community. Although long or 
short sleep is associated with an increased risk of some health outcomes, there is no rigorous evidence that lengthening or shortening sleep duration can lead to a smaller frequency of these outcomes.

In conclusion, abnormal duration or quality of sleep was significantly associated with an extensive range of adverse healthrelated outcomes. Based on our umbrella review, although 36 studies explored 36 unique associations, the highly suggestive evidence only supported that long sleep duration was associated with an increased risk of all-cause mortality. The relationship between abnormal duration or quality of sleep and other outcomes could be genuine, but there is still limited evidence for them. Overall, this article assessed the associations between duration and quality of sleep and health outcomes based on previous studies, which is helpful for identifying at-risk groups and developing prevention strategies to counteract the effect of sleep discrepancies. Abnormal duration or quality of sleep is harmful to human health, but further high-quality prospective studies and better designed trials are needed to generate definite conclusions.

\section{DATA AVAILABILITY STATEMENT}

The original contributions presented in the study are included in the article/Supplementary Material, further inquiries can be directed to the corresponding authors.

\section{REFERENCES}

1. Foundation NS. How Much Sleep Do We Really Need? (2018). Available online at: https://www.sleepfoundation.org/how-sleep-works/how-muchsleep-do-we-really-need (accessed June 27, 2018).

2. Roncoroni J, Dong Y, Owen J, Wippold G. The association of sleep duration and feeling rested with health in US Hispanic women. Sleep Med. (2021) 83:54-62. doi: 10.1016/j.sleep.2021.03.044

3. Park S, Lee S, Kim Y, Lee Y, Kang MW, Kim K. Short or long sleep duration and CKD: a Mendelian Randomization Study. J Am Soc Nephrol. (2021) 31:2937-47. doi: 10.1681/ASN.2020050666

4. Blair LM, Porter K, Leblebicioglu B, Christian LM. Poor sleep quality and associated inflammation predict preterm birth: heightened risk among African Americans. Sleep. (2015) 38:1259-67. doi: 10.5665/sleep.4904

5. Aromataris E, Fernandez R, Godfrey CM, Holly C, Khalil H, Tungpunkom P. Summarizing systematic reviews: methodological development, conduct and reporting of an umbrella review approach. Int J Evid Based Healthc. (2015) 13:132-40. doi: 10.1097/XEB.0000000000000055

6. Moher D, Liberati A, Tetzlaff J, Altman DG. Preferred reporting items for systematic reviews and meta-analyses: the PRISMA statement. PLoS Med. (2009) 6:e1000097. doi: 10.1371/journal.pmed.1000097

7. Stroup DF, Berlin JA, Morton SC, Olkin I, Williamson GD, Rennie D. Metaanalysis of observational studies in epidemiology: a proposal for reporting. Meta-analysis of observational studies in epidemiology (MOOSE) group. JAMA. (2000) 283:2008-12. doi: 10.1001/jama.283.15.2008

8. Liu FH, Liu C, Gong TT, Gao S, Sun H, Jiang YT. Dietary inflammatory index and health outcomes: an umbrella review of systematic review and meta-analyses of observational studies. Front Nutr. (2021) 8:647122. doi: 10.3389/fnut.2021.647122

9. Marx W, Veronese N, Kelly JT, Smith L, Hockey M, Collins S, et al. The dietary inflammatory index and human health: an umbrella review of meta-analyses of observational studies. Adv Nutr. (2021) 12:1681-90. doi: 10.1093/advances/nmab037

\section{AUTHOR CONTRIBUTIONS}

T-TG and Q-JW contributed to the study design. CG and X-YL conducted the literature search. JG, J-LL, X-YL, F-HL, and MZ extracted the data and conducted the analyses. CG, JG, T-TG, J-LL, Y-TS, and Y-HZ wrote the first draft of the manuscript and edited the manuscript. All authors read and approved the final manuscript and accept responsibility for the integrity of the data analyzed.

\section{FUNDING}

This work was supported by the National Key R\&D Program of China (No. 2017YFC0907403 to Y-HZ), Natural Science Foundation of China (Nos. 82073647 and 81602918 to Q-JW and No. 82103914 to T-TG), LiaoNing Revitalization Talents Program (No. XLYC1907102 to Q-JW), Shenyang High-level Innovative Talents Support Program (No. RC190484 to Q-JW), and 345 Talent Project of Shengjing Hospital of China Medical University (Q-JW and T-TG).

\section{SUPPLEMENTARY MATERIAL}

The Supplementary Material for this article can be found online at: https://www.frontiersin.org/articles/10.3389/fmed. 2021.813943/full\#supplementary-material

10. Sun H, Gong TT, Xia Y, Wen ZY, Zhao LG, Zhao YH, et al. Diet and ovarian cancer risk: an umbrella review of systematic reviews and meta-analyses of cohort studies. Clin. Nutr. (2021) 40:1682-90. doi: 10.1016/j.clnu.2020.11.032

11. Chinn S. A simple method for converting an odds ratio to effect size for use in meta-analysis. Stat Med. (2000) 19:3127-31. doi: 10.1002/10970258(20001130)19:22<3127::AID-SIM784>3.0.CO;2-M

12. Lau J, Ioannidis JP, Schmid CH. Quantitative synthesis in systematic reviews. Ann Intern Med. (1997) 127:820-6. doi: 10.7326/0003-4819-127-9-199711010-00008

13. Higgins JP, Thompson SG, Deeks JJ, Altman DG. Measuring inconsistency in meta-analyses. BMJ. (2003) 327:557-60. doi: 10.1136/bmj.327.7414.557

14. Ioannidis JP, Patsopoulos NA, Evangelou E. Uncertainty in heterogeneity estimates in meta-analyses. BMJ. (2007) 335:914-6. doi: $10.1136 / \mathrm{bmj} .39343 .408449 .80$

15. Riley RD, Higgins JP, Deeks JJ. Interpretation of random effects metaanalyses. BMJ. (2011) 342:d549. doi: 10.1136/bmj.d549

16. Egger M, Davey Smith G, Schneider M, Minder C. Bias in metaanalysis detected by a simple, graphical test. BMJ. (1997) 315:629-34. doi: 10.1136/bmj.315.7109.629

17. Sterne JA, Sutton AJ, Ioannidis JP, Terrin N, Jones DR, Lau J. Recommendations for examining and interpreting funnel plot asymmetry in meta-analyses of randomised controlled trials. BMJ. (2011) 343:d4002. doi: 10.1136/bmj.d4002

18. Ioannidis JP, Trikalinos TA. An exploratory test for an excess of significant findings. Clin Trials. (2007) 4:245-53. doi: 10.1177/1740774507079441

19. Tsilidis KK, Panagiotou OA, Sena ES, Aretouli E, Evangelou E, Howells DW. Evaluation of excess significance bias in animal studies of neurological diseases. PLoS Biol. (2013) 11:e1001609. doi: 10.1371/journal.pbio.1001609

20. da Costa BR, Rutjes AW, Johnston BC, Reichenbach S, Nüesch E, Tonia T, et al. Methods to convert continuous outcomes into odds ratios of treatment response and numbers needed to treat: meta-epidemiological study. Int J Epidemiol. (2012). 41:1445-59. doi: 10.1093/ije/dys124 
21. Shea BJ, Grimshaw JM, Wells GA, Boers M, Andersson N, Hamel C. Development of AMSTAR: a measurement tool to assess the methodological quality of systematic reviews. BMC Med Res Methodol. (2007) 7:10. doi: 10.1186/1471-2288-7-10

22. Kalliala I, Markozannes G, Gunter MJ, Paraskevaidis E, Gabra H, Mitra A. Obesity and gynaecological and obstetric conditions: umbrella review of the literature. BMJ. (2017) 359:j4511. doi: 10.1136/bmj.j4511

23. Chokesuwattanaskul R, Thongprayoon C, Sharma K, Congrete S, Tanawuttiwat T, Cheungpasitporn W. Associations of sleep quality with incident atrial fibrillation: a meta-analysis. Intern Med J. (2018) 48:964-72. doi: 10.1111/imj.13764

24. Yang X, Chen H, Pan LiS, Jia L, C. Association of sleep duration with the morbidity and mortality of coronary artery disease: a metaanalysis of prospective studies. Heart Lung Circ. (2015) 24:1180-90. doi: 10.1016/j.hlc.2015.08.005

25. Yin J, Jin X, Shan Z, Li S, Huang H, Li P, et al. Relationship of sleep duration with all-cause mortality and cardiovascular events: a systematic review and dose-response meta-analysis of prospective cohort studies. J Am Heart Assoc. (2017) 6:e005947. doi: 10.1161/JAHA.117.005947

26. Meng L, Zheng Y, Hui R. The relationship of sleep duration and insomnia to risk of hypertension incidence: a meta-analysis of prospective cohort studies. Hypertens Res. (2013) 36:985-95. doi: 10.1038/hr.2013.70

27. Ren LiH, Wu Y, Zhao Y, X. Correlation between sleep duration and hypertension: a dose-response meta-analysis. J Hum Hypertens. (2019) 33:218-28. doi: 10.1038/s41371-018-0135-1

28. Kwok CS, Kontopantelis E, Kuligowski G, Gray M, Muhyaldeen A, Gale CP. Self-reported sleep duration and quality and cardiovascular disease and mortality: a dose-response meta-analysis. J Am Heart Assoc. (2018) 7:e008552. doi: 10.1161/JAHA.118.008552

29. Fan L, Xu W, Cai Y, Hu Y, Wu C. Sleep duration and the risk of dementia: a systematic review and meta-analysis of prospective cohort studies. J Am Med Directors Assoc. (2019). 20:1480-7.e5. doi: 10.1016/j.jamda.2019.06.009

30. Wu L, Sun D, Tan Y. A systematic review and dose-response meta-analysis of sleep duration and the occurrence of cognitive disorders. Sleep Breath. (2018) 22:805-14. doi: 10.1007/s11325-017-1527-0

31. Liang Y, Qu LB, Liu H. Non-linear associations between sleep duration and the risks of mild cognitive impairment/dementia and cognitive decline: a dose-response meta-analysis of observational studies. Aging Clin Exp Res. (2019) 31:309-20. doi: 10.1007/s40520-018-1005-y

32. Leng Y, Cappuccio FP, Wainwright NW, Surtees PG, Luben R, Brayne C. Sleep duration and risk of fatal and nonfatal stroke: a prospective study and meta-analysis. Neurology. (2015) 84:1072-9. doi: 10.1212/WNL.0000000000001371

33. He Q, Sun H, Wu X, Zhang P, Dai H, Ai C, et al. Sleep duration and risk of stroke: a dose-response meta-analysis of prospective cohort studies. Sleep Med. (2017) 32:66-74. doi: 10.1016/j.sleep.2016.12.012

34. Holliday EG, Magee CA, Kritharides L, Banks E, Attia J. Short sleep duration is associated with risk of future diabetes but not cardiovascular disease: a prospective study and meta-analysis. PLoS ONE. (2013) 8:e82305. doi: 10.1371/journal.pone.0082305

35. Cappuccio FP, D’Elia L, Strazzullo P, Miller MA. Quantity and quality of sleep and incidence of type 2 diabetes: a systematic review and meta-analysis. Diabetes Care. (2010) 33:414-20. doi: 10.2337/dc09-1124

36. Shan Z, Ma H, Xie M, Yan P, Guo Y, Bao W. Sleep duration and risk of type 2 diabetes: a meta-analysis of prospective studies. Diabetes Care. (2015) 38:529-37. doi: 10.2337/dc14-2073

37. Cai LiY, Ling S, Mi Y, Fan S, Zhong CY, et al. Association between total sleep time and all cancer mortality: non-linear dose-response meta-analysis of cohort studies. Sleep Med. (2019) 60:211-8. doi: 10.1016/j.sleep.2019. 03.026

38. Stone CR, Haig TR, Fiest KM, McNeil J, Brenner DR, Friedenreich CM. The association between sleep duration and cancer-specific mortality: a systematic review and meta-analysis. Cancer Causes Control. (2019). 30:50125. doi: 10.1007/s10552-019-01156-4

39. Cappuccio FP, D’Elia L, Strazzullo P, Miller MA. Sleep duration and all-cause mortality: a systematic review and meta-analysis of prospective studies. Sleep. (2010) 33:585-92. doi: 10.1093/sleep/33.5.585
40. Gallicchio L, Kalesan B. Sleep duration and mortality: a systematic review and meta-analysis. J Sleep Res. (2009) 18:148-58. doi: 10.1111/j.1365-2869.2008.00732.x

41. Liu R, Wu S, Zhang B, Guo M, Zhang Y. The association between sleep duration and prostate cancer: A systematic review and meta-analysis. Medicine. (2020) 99:e21180. doi: 10.1097/MD.0000000000021180

42. Wang LiW, Cao D, Yin S, Gong X, Gan YY, et al. Sleep duration and risk of stroke events and stroke mortality: a systematic review and metaanalysis of prospective cohort studies. Int J Cardiol. (2016) 223:870-6. doi: 10.1016/j.ijcard.2016.08.302

43. Wong ATY, Heath AK, Tong TYN, Reeves GK, Floud S, Beral V, et al. Sleep duration and breast cancer incidence: results from the Million Women Study and meta-analysis of published prospective studies. Sleep. (2021) 44:166. doi: 10.1093/sleep/zsaa166

44. Lu Y, Tian N, Yin J, Shi Y, Huang Z. Association between sleep duration and cancer risk: a meta-analysis of prospective cohort studies. PLoS ONE. (2013) 8:e74723. doi: 10.1371/journal.pone.0074723

45. Zhao H, Yin JY, Yang WS, Qin Q, Shi Li TT. Sleep duration and cancer risk: a systematic review and meta-analysis of prospective studies. Asian Pac J Cancer Prev. (2013) 14:7509-15. doi: 10.7314/APJCP.2013.14.12.7509

46. Miller MA, Bates S, Cappuccio Ji C. Systematic review and meta-analyses of the relationship between short sleep and incidence of obesity and effectiveness of sleep interventions on weight gain in preschool children. Obes Rev. (2021) 22:e13113. doi: 10.1111/obr.13113

47. Wu Y, Gong Q, Zou Z, Zhang LiH. Short sleep duration and obesity among children: A systematic review and meta-analysis of prospective studies. Obes Res Clin Pract. (2017) 11:140-50. doi: 10.1016/j.orcp.2016.05.005

48. Ruan H, Xun P, Cai W, He K, Tang Q. Habitual sleep duration and risk of childhood obesity: systematic review and dose-response meta-analysis of prospective cohort studies. Sci Rep. (2015) 5:16160. doi: 10.1038/srep16160

49. Fatima Y, Doi SA, Mamun AA. Longitudinal impact of sleep on overweight and obesity in children and adolescents: a systematic review and biasadjusted meta-analysis. Obes Rev. (2015) 16:137-49. doi: 10.1111/obr.12245

50. Miller MA, Kruisbrink M, Wallace J, Ji C, Cappuccio FP. Sleep duration and incidence of obesity in infants, children, and adolescents: a systematic review and meta-analysis of prospective studies. Sleep. (2018) 41:18. doi: 10.1093/sleep/zsy018

51. Zhai L, Zhang H, Zhang D. Sleep duration and depression among adults: a meta-analysis of prospective studies. Depress Anxiety. (2015) 32:664-70. doi: $10.1002 /$ da.22386

52. Kruisbrink M, Robertson W, Miller JiC, Geleijnse MA, Cappuccio JM. Association of sleep duration and quality with blood lipids: a systematic review and meta-analysis of prospective studies. BMJ Open. (2017) 7:e018585. doi: 10.1136/bmjopen-2017-018585

53. Zhang X, Zhang R, Cheng L, Wang Y, Ding X, Fu J. The effect of sleep impairment on gestational diabetes mellitus: a systematic review and meta-analysis of cohort studies. Sleep Med. (2020) 74:267-77. doi: 10.1016/j.sleep.2020.05.014

54. Xu YH, Shi L, Bao YP, Chen SJ, Shi J, Zhang RL. Association between sleep duration during pregnancy and gestational diabetes mellitus: a metaanalysis. Sleep Med. (2018) 52:67-74. doi: 10.1016/j.sleep.2018.07.021

55. Cappuccio FP, Cooper D, D’Elia L, Strazzullo P, Miller MA. Sleep duration predicts cardiovascular outcomes: a systematic review and meta-analysis of prospective studies. Eur Heart J. (2011) 32:1484-92. doi: 10.1093/eurheartj/ehr007

56. Anothaisintawee T, Reutrakul S, Van Cauter E, Thakkinstian A. Sleep disturbances compared to traditional risk factors for diabetes development: systematic review and meta-analysis. Sleep Med Rev. (2016) 30:11-24. doi: 10.1016/j.smrv.2015.10.002

57. Hao G, Zhu B, Wang LiY, Li P, Hou L, L. Sleep quality and disease activity in patients with inflammatory bowel disease: a systematic review and meta-analysis. Sleep Med. (2020) 75:301-8. doi: 10.1016/j.sleep.2020. 08.032

58. Wang L, Jin F. Association between maternal sleep duration and quality, and the risk of preterm birth: a systematic review and metaanalysis of observational studies. BMC Preg Childbirth. (2020) 20:125. doi: 10.1186/s12884-020-2814-5 
59. Bellavia A, Åkerstedt T, Bottai M, Wolk A, Orsini N. Sleep duration and survival percentiles across categories of physical activity. Am J Epidemiol. (2014) 179:484-91. doi: 10.1093/aje/kwt280

60. Magee CA, Holliday EG, Attia J, Kritharides L, Banks E. Investigation of the relationship between sleep duration, all-cause mortality, and preexisting disease. Sleep Med. (2013) 14:591-6. doi: 10.1016/j.sleep.2013.02.002

61. Xiao Q, Keadle SK, Hollenbeck AR, Matthews CE. Sleep duration and total and cause-specific mortality in a large US cohort: interrelationships with physical activity, sedentary behavior, and body mass index. Am J Epidemiol. (2014) 180:997-1006. doi: 10.1093/aje/kwu222

62. Yeo Y, Ma SH, Park SK, Chang SH, Shin HR, Kang D. A prospective cohort study on the relationship of sleep duration with all-cause and disease-specific mortality in the Korean Multi-center Cancer Cohort study. J Prev Med Public Health. (2013) 46:271-81. doi: 10.3961/jpmph.2013.46.5.271

63. Chen HC, Su TP, Chou P. A nine-year follow-up study of sleep patterns and mortality in community-dwelling older adults in Taiwan. Sleep. (2013) 36:1187-98. doi: 10.5665/sleep.2884

64. Stranges S, Dorn JM, Shipley MJ, Kandala NB, Trevisan M, Miller MA. Correlates of short and long sleep duration: a cross-cultural comparison between the United Kingdom and the United States: the Whitehall II Study and the Western New York Health Study. Am J Epidemiol. (2008) 168:135364. doi: 10.1093/aje/kwn337

65. Grandner MA, Buxton OM, Jackson N, Sands-Lincoln M, Pandey A, JeanLouis G. Extreme sleep durations and increased C-reactive protein: effects of sex and ethnoracial group. Sleep. (2013) 36:769-79e. doi: 10.5665/sleep.2646

66. Grandner MA, Sands-Lincoln MR, Pak VM, Garland SN. Sleep duration, cardiovascular disease, and proinflammatory biomarkers. Nat Sci Sleep. (2013) 5:93-107. doi: 10.2147/NSS.S31063

67. Patel SR, Zhu X, Storfer-Isser A, Mehra R, Jenny NS, Tracy R. Sleep duration and biomarkers of inflammation. Sleep. (2009) 32:200-4. doi: 10.1093/sleep/32.2.200

68. Prather AA, Vogelzangs N, Penninx BW. Sleep duration, insomnia, and markers of systemic inflammation: results from the Netherlands Study of Depression and Anxiety (NESDA). J Psychiatr Res. (2015) 60:95-102. doi: 10.1016/j.jpsychires.2014.09.018

69. Abe T, Aoki T, Yata S, Okada M. Sleep duration is significantly associated with carotid artery atherosclerosis incidence in a Japanese population. Atherosclerosis. (2011) 217:509-13. doi: 10.1016/j.atherosclerosis.2011.02.029

70. Khawaja O, Sarwar A, Albert CM, Gaziano JM, Djoussé L. Sleep duration and risk of atrial fibrillation (from the Physicians' Health Study). Am J Cardiol. (2013) 111:547-51. doi: 10.1016/j.amjcard.2012.10.038

71. Ramos AR, Dong C, Rundek T, Elkind MS, Boden-Albala B, Sacco RL. Sleep duration is associated with white matter hyperintensity volume in older adults: the Northern Manhattan Study. J Sleep Res. (2014) 23:524-30. doi: 10.1111 /jsr. 12177

72. Wolf PA, Dawber TR, Thomas HE, Jr., Kannel WB. Epidemiologic assessment of chronic atrial fibrillation and risk of stroke: the Framingham study. Neurology. (1978) 28:973-7. doi: 10.1212/WNL.28.10.973

73. Wolff B, Völzke H, Schwahn C, Robinson D, Kessler C, John U. Relation of self-reported sleep duration with carotid intima-media thickness in a general population sample. Atherosclerosis. (2008) 196:727-32. doi: 10.1016/j.atherosclerosis.2006.12.023

74. Kakizaki M, Kuriyama S, Nakaya N, Sone T, Nagai M, Sugawara Y. Long sleep duration and cause-specific mortality according to physical function and self-rated health: the Ohsaki Cohort Study. J Sleep Res. (2013) 22:209-16. doi: 10.1111/j.1365-2869.2012.01053.x

75. Pan A, De Silva DA, Yuan JM, Koh WP. Sleep duration and risk of stroke mortality among Chinese adults: Singapore Chinese health study. Stroke. (2014). 45:1620-5. doi: 10.1161/STROKEAHA.114.005181

76. Qureshi AI, Giles WH, Croft JB, Bliwise DL. Habitual sleep patterns and risk for stroke and coronary heart disease: a 10-year follow-up from NHANES I. Neurology. (1997) 48:904-11. doi: 10.1212/WNL.48.4.904

77. Fischer AQ, Chaudhary BA, Taormina MA, Akhtar B. Intracranial hemodynamics in sleep apnea. Chest. (1992) 102:1402-6. doi: $10.1378 /$ chest.102.5.1402

78. Jennum P, Børgesen SE. Intracranial pressure and obstructive sleep apnea. Chest. (1989) 95:279-83. doi: 10.1378/chest.95.2.279
79. Daly JAGR, Miller B, Garret K. Impaired awake cerebral perfusion in sleep apnea [abstract]. Am Rev Respir Dis. (1990) 141:A376.

80. Klingelhöfer J, Hajak G, Sander D, Schulz-Varszegi M, Rüther E, Conrad B. Assessment of intracranial hemodynamics in sleep apnea syndrome. Stroke. (1992) 23:1427-33. doi: 10.1161/01.STR.23.10.1427

81. Ge B, Guo X. Short and long sleep durations are both associated with increased risk of stroke: a meta-analysis of observational studies. Int J Stroke. (2015) 10:177-84. doi: 10.1111/ijs.12398

82. Titova OE, Michaëlsson K, Larsson SC. Sleep duration and stroke: prospective cohort study and mendelian randomization analysis. Stroke. (2020) 51:3279-85. doi: 10.1161/STROKEAHA.120.029902

83. Leng Y, Wainwright NW, Cappuccio FP, Surtees PG, Luben R, Wareham N. Self-reported sleep patterns in a British population cohort. Sleep Med. (2014) 15:295-302. doi: 10.1016/j.sleep.2013.10.015

84. Petrov ME, Howard G, Grandner MA, Kleindorfer D, Molano JR, Howard VJ. Sleep duration and risk of incident stroke by age, sex, and race: the REGARDS study. Neurology. (2018) 91:e1702-e9. doi: 10.1212/WNL.0000000000006424

85. Khan H, Kella D, Kunutsor SK, Savonen K, Laukkanen JA. Sleep duration and risk of fatal coronary heart disease, sudden cardiac death, cancer death, and all-cause mortality. Am J Med. (2018) 131:1499-505.e2. doi: 10.1016/j.amjmed.2018.07.010

86. Grandner MA, Drummond SP. Who are the long sleepers? Towards an understanding of the mortality relationship. Sleep Med Rev. (2007) 11:34160. doi: 10.1016/j.smrv.2007.03.010

87. Hare DL, Toukhsati SR, Johansson P, Jaarsma T. Depression and cardiovascular disease: a clinical review. Eur Heart J. (2014) 35:1365-72. doi: 10.1093/eurheartj/eht462

88. Spiegel K, Tasali E, Penev P, Van Cauter E. Brief communication: sleep curtailment in healthy young men is associated with decreased leptin levels, elevated ghrelin levels, and increased hunger and appetite. Ann Intern Med. (2004) 141:846-50. doi: 10.7326/0003-4819-141-11-200412070-00008

89. Taheri S, Lin L, Austin D, Young T, Mignot E. Short sleep duration is associated with reduced leptin, elevated ghrelin, and increased body mass index. PLoS Med. (2004) 1:e62. doi: 10.1371/journal.pmed.0010062

90. Cedernaes J, Fanelli F, Fazzini A, Pagotto U, Broman JE, Vogel H. Sleep restriction alters plasma endocannabinoids concentrations before but not after exercise in humans. Psychoneuroendocrinology. (2016) 74:258-68. doi: 10.1016/j.psyneuen.2016.09.014

91. Taheri S. The link between short sleep duration and obesity: we should recommend more sleep to prevent obesity. Arch Dis Child. (2006) 91:881-4. doi: 10.1136/adc.2005.093013

92. Chen X, Beydoun MA, Wang Y. Is sleep duration associated with childhood obesity? A systematic review and meta-analysis. Obesity. (2008) 16, 265-74. doi: $10.1038 /$ oby. 2007.63

93. Spiegel K, Tasali E, Leproult R, Van Cauter E. Effects of poor and short sleep on glucose metabolism and obesity risk. Nat Rev Endocrinol. (2009) 5:253-61. doi: 10.1038/nrendo.2009.23

94. Beccuti G, Pannain S. Sleep and obesity. Curr Opin Clin Nutr Metab Care. (2011) 14:402-12. doi: 10.1097/MCO.0b013e3283479109

95. Broussard JL, Ehrmann DA, Van Cauter E, Tasali E, Brady MJ. Impaired insulin signaling in human adipocytes after experimental sleep restriction: a randomized, crossover study. Ann Intern Med. (2012) 157:549-57. doi: 10.7326/0003-4819-157-8-201210160-00005

96. Drager LF, Togeiro SM, Polotsky VY, Lorenzi-Filho G. Obstructive sleep apnea: a cardiometabolic risk in obesity and the metabolic syndrome. J Am Coll Cardiol. (2013) 62:569-76. doi: 10.1016/j.jacc.2013.05.045

97. Miller MA, Cappuccio FP. Inflammation, sleep, obesity and cardiovascular disease. Curr Vasc Pharmacol. (2007) 5:93-102. doi: 10.2174/157016107780368280

98. Möller-Levet CS, Archer SN, Bucca G, Laing EE, Slak A, Kabiljo R. Effects of insufficient sleep on circadian rhythmicity and expression amplitude of the human blood transcriptome. Proc Natl Acad Sci USA. (2013) 110:E1132-41. doi: 10.1073/pnas.1217154110

99. Taveras EM, Rifas-Shiman SL, Bub KL, Gillman MW, Oken E. Prospective study of insufficient sleep and neurobehavioral functioning among schoolage children. Acad Pediatr. (2017) 17:625-32. doi: 10.1016/j.acap.2017. 02.001 
100. Lee SWH, Ng KY, Chin WK. The impact of sleep amount and sleep quality on glycemic control in type 2 diabetes: a systematic review and meta-analysis. Sleep Med Rev. (2017) 31:91-101. doi: 10.1016/j.smrv.2016.02.001

101. Boyko EJ, Seelig AD, Jacobson IG, Hooper TI, Smith B, Smith TC. Sleep characteristics, mental health, and diabetes risk: a prospective study of US military service members in the Millennium Cohort Study. Diab Care. (2013) 36:3154-61. doi: 10.2337/DC13-0042

102. Lai YJ, Lin CL, Lin MC, Lee ST, Sung FC, Chang YJ. Population-based cohort study on the increase in the risk for type 2 diabetes mellitus development from nonapnea sleep disorders. Sleep Med. (2013) 14:913-8. doi: 10.1016/j.sleep.2013.03.024

103. Zhong C, Chen R, Zhou X, Xu S, Cui LiQ. Poor sleep during early pregnancy increases subsequent risk of gestational diabetes mellitus. Sleep Med. (2018) 46:20-5. doi: 10.1016/j.sleep.2018.02.014

104. Rafalson L, Donahue RP, Stranges S, Lamonte MJ, Dmochowski J, Dorn J. Short sleep duration is associated with the development of impaired fasting glucose: the Western New York Health Study. Ann Epidemiol. (2010) 20:883-9. doi: 10.1016/j.annepidem.2010.05.002

105. Spiegel K, Leproult R, Van Cauter E. Impact of sleep debt on metabolic and endocrine function. Lancet. (1999) 354:1435-9. doi: 10.1016/S0140-6736(99)01376-8

106. Thomas M, Sing H, Belenky G, Holcomb H, Mayberg H, Dannals R. Neural basis of alertness and cognitive performance impairments during sleepiness. I Effects of $24 \mathrm{~h}$ of sleep deprivation on waking human regional brain activity. J Sleep Res. (2000) 9:335-52. doi: 10.1046/j.1365-2869.2000.00225.x

107. Grassi, G. Dell'Oro R, Quarti-Trevano F, Scopelliti F, Seravalle G, Paleari F, et al. (2005). Neuroadrenergic and reflex abnormalities in patients with metabolic syndrome. Diabetologia. 48:1359-65. doi: 10.1007/s00125-005-1798-Z

108. Tentolouris N, Argyrakopoulou G, Katsilambros N. Perturbed autonomic nervous system function in metabolic syndrome. Neuromolecular Med. (2008) 10:169-78. doi: 10.1007/s12017-008-8022-5

109. Buxton OM, Pavlova M, Reid EW, Wang W, Simonson DC, Adler GK. Sleep restriction for 1 week reduces insulin sensitivity in healthy men. Diabetes. (2010) 59:2126-33. doi: 10.2337/db09-0699

110. Leproult R, Van Cauter E. Role of sleep and sleep loss in hormonal release and metabolism. Endocr Dev. (2010) 17:11-21. doi: 10.1159/000262524

111. Vgontzas AN, Mastorakos G, Bixler EO, Kales A, Gold PW, Chrousos GP. Sleep deprivation effects on the activity of the hypothalamic-pituitary-adrenal and growth axes: potential clinical implications. Clin Endocrinol. (1999) 51:205-15. doi: 10.1046/j.1365-2265.1999.00763.x

112. Esposito K, Nappo F, Marfella R, Giugliano G, Giugliano F, Ciotola M. Inflammatory cytokine concentrations are acutely increased by hyperglycemia in humans: role of oxidative stress. Circulation. (2002) 106:2067-72. doi: 10.1161/01.CIR.0000034509.14906.AE

113. Larkin EK, Rosen CL, Kirchner HL, Storfer-Isser A, Emancipator JL, Johnson NL. Variation of C-reactive protein levels in adolescents: association with sleep-disordered breathing and sleep duration. Circulation. (2005) 111:197884. doi: 10.1161/01.CIR.0000161819.76138.5E

114. Tasali E, Leproult R, Ehrmann DA, Van Cauter E. Slow-wave sleep and the risk of type 2 diabetes in humans. Proc Natl Acad Sci USA. (2008) 105:1044-9. doi: 10.1073/pnas.0706446105

115. Broussard J, Brady MJ. The impact of sleep disturbances on adipocyte function and lipid metabolism. Best practice and research. Clin Endocrinol Metab. (2010) 24:763-73. doi: 10.1016/j.beem.2010.08.007

116. Mollayeva T, Thurairajah P, Burton K, Mollayeva S, Shapiro CM, Colantonio A. The Pittsburgh sleep quality index as a screening tool for sleep dysfunction in clinical and non-clinical samples: a systematic review and meta-analysis. Sleep Med Rev. (2016) 25:52-73. doi: 10.1016/j.smrv.2015. 01.009

Conflict of Interest: The authors declare that the research was conducted in the absence of any commercial or financial relationships that could be construed as a potential conflict of interest.

Publisher's Note: All claims expressed in this article are solely those of the authors and do not necessarily represent those of their affiliated organizations, or those of the publisher, the editors and the reviewers. Any product that may be evaluated in this article, or claim that may be made by its manufacturer, is not guaranteed or endorsed by the publisher.

Copyright (C) 2022 Gao, Guo, Gong, Lv, Li, Liu, Zhang, Shan, Zhao and Wu. This is an open-access article distributed under the terms of the Creative Commons Attribution License (CC BY). The use, distribution or reproduction in other forums is permitted, provided the original author(s) and the copyright owner(s) are credited and that the original publication in this journal is cited, in accordance with accepted academic practice. No use, distribution or reproduction is permitted which does not comply with these terms. 